\title{
Entropic solvation force between surfaces modified by grafted chains: a density functional approach
}

\author{
O. Pizid 1 , S. Sokołowsk² \\ 1 Instituto de Química de la UNAM, Coyoacán 04510, México \\ 2 Department for the Modeling of Physico-Chemical Processes, Maria Curie-Skłodowska University, \\ 20-031 Lublin, Poland
}

Received October 21, 2009, in final form January 27, 2010

\begin{abstract}
The behavior of a hard sphere fluid in slit-like pores with walls modified by grafted chain molecules composed of hard sphere segments is studied using density functional theory. The chains are grafted to opposite walls via terminating segments forming pillars. The effects of confinement and of "chemical" modification of pore walls on the entropic solvation force are investigated in detail. We observe that in the absence of adsorbed fluid the solvation force is strongly repulsive for narrow pores and attractive for wide pores. In the presence of adsorbed fluid both parts of the curve of the solvation force may develop oscillatory behavior dependent on the density of pillars, the number of segments and adsorption conditions. Also, the size ratio between adsorbed fluid species and chain segments is of importance for the development of oscillations. The choice of these parameters is crucial for efficient manipulation of the solvation force as desired for pores of different width.
\end{abstract}

Key words: density functional approach, grafted chains, solvation force, density profiles

PACS: 68.08.-p, 68.15.+e, 82.35.Gh, 68.43.-h

\section{Introduction}

Thin fluid films confined by solid surfaces are of much interest for basic research and several applications. The microscopic structure, thermodynamic, electric and other properties of such systems have been extensively studied in experiments, computer simulations and using various theoretical methods. One of the important properties of this type of systems is the solvation force, i.e. the force acting between solid surfaces due to the intervening fluid between them. Experiments using surface force apparatus yield the solvation force that indirectly describes the fluid structure in confined films [1-4]. Several important conclusions for numerous applications have been made by analyzing experimental results for the solvation force.

The solvation force is determined by different factors, e.g. it is effected by the properties of molecules of confined fluid [5-9]. Simple fluids, associating and hydrogen-bonded fluids, fluids of complex molecules as well as some mixtures and solutions were investigated experimentally in this aspect. On the other hand, computer simulation and theoretical methods have been extensively applied to the study of the solvation force [10-16]. The dependence of solvation force on the characteristics of confining solid substrates, namely the shape of the surface, its in-plane symmetry, energetic and geometric heterogeneity, was investigated in many publications, see e.g. [17 21]. In the last decade, much research has been focused on synthetic and chemically modified substrates for specific applications 22 26].

From a general theoretical perspective, one of the powerful and popular tools of studying inhomogeneous fluids is the density functional theory. Modern versions of this theory are capable of accurately describing the microscopic structure, thermodynamic properties and phase behavior of confined fluid systems. This is proved by comparison of theoretical results with computer simulation data. In particular, investigations of the solvation force for some model fluid systems by means of density functional approaches have been undertaken [27 29]. In the recent works, Cao and Wu [30], as well as Jiang et al. 31] examined the solvation force between plane walls in the presence of 
telechelic polymers in the pore space. We give a few details regarding their modelling in order to stress the differences between that approach and the present study. The telechelic polymers were considered [30, 31] to be built up of the jointed tangent-sphere chains with "sticky" ends adhered to a solid surface. It was assumed that the terminating segments of each chain were attracted to the hard wall via narrow square well potential. On the other hand, the "sticky" segments themselves interacted via square well attractive potential. The solvent effects were totally neglected; the solvent was treated as a continuum without any specification. A polymer density functional theory was used to obtain theoretical predictions. Excluded volume effects in the excess free energy and the effects of chain connectivity were considered using the fundamental measure and the first-order perturbation theories, respectively [32]. The attractive interactions between terminating segments were accounted for at the level of a mean field theory. The solvation force between substrates coming from the presence of telechelic polymers and their grafting was explored for a model permitting the formation of brushes at each pore wall (due to a single adhesive segment on chain molecules) and a model in which each surface was covered by loops (due to two adhesive segments per chain). Most recently, the effects of energetic parameters characterizing attractive interactions in the model, in addition to the density changes of grafted species were studied within the same theoretical framework [33, 34].

In the present work we use a methodologically similar approach, however, with some modifications described below. Our principal objective is to investigate the solvation force for a model system that explicitly includes a simple fluid confined by solid walls. These walls are modified by grafted chain molecules. Each chain molecule in our study consists of segments of equal diameter (homopolymer), all the molecules being pinned to the opposite walls of a pore through the first and the last segment forming flexible pillars. The solvation force comes from the presence of chain particles and fluid species. The fluid species are taken into account explicitly, in contrast to the model by $\mathrm{Cao}$ and $\mathrm{Wu} 30,31$. However, to elucidate the underlying phenomena in such a class of systems, at this stage of investigation we restrict ourselves to the model in which only the repulsive forces are taken into account. Namely, the fluid and segments of chain species are hard spheres. The solid substrates solely provide geometric confinement. Thus, we are able to describe the underlying entropic contributions to the solvation force between pore walls joined by pillars.

Entropic effects in confined fluids are common. They arise either due to a molecular shape or internal architecture of molecules or, on the other hand, can be of combined nature, namely involving the former factors and specific form of interaction between complex molecules and substrates. Entropic effects can lead to various phase transformations in confined fluids in the absence of attractive interactions determining energetic aspects of adsorption and surface phase transitions. Our setup seems to be simple, but permits various physically interesting extensions and generalizations. Namely, one can extend the present techniques to explore the adsorption of mixtures in which entropic effects under confinement may induce different phase separations, e.g. nonadditive hard sphere mixtures, hard sphere mixtures with rather big difference of diameters of particles belonging to different components, the Widow-Rowlinson model, the Asakura-Oosawa model for colloid-polymer mixtures and some other complex fluids. Also, it is of interest to formulate the problem of swelling at the level of present modelling. However, before comprehensively studying such models under confinement, one need to have a rather complete understanding of a comparatively simpler system in question. Even in the present formulation, the model is characterized by various parameters. Investigation of the role of each of them is one of our primary objectives. As for the subsystem of chains, most important is to capture the effect of grafted density and length of chains that form pillars between walls. On the other hand, the density of fluid species in the pore is not of less importance. Finally, the ratio of diameters of chain segments and of fluid particles affects adsorption and microscopic structure, i.e. both the distribution of fluid species and the way segments of chains accommodate in the pore.

The theoretical approach has been described in detail in our recent works [35 40]. Therefore, hereinafter we will give only some essential ingredients. Our approach is based on the method developed by $\mathrm{Yu}$ and $\mathrm{Wu}[32$ and extended later by us to describe a particular mixture of spherical 
and chain molecules in which some chains are pinned to the walls. We expect that the insights obtained in the present study may be useful in considering the novel setups for nano-systems and devices with desired interfacial properties.

\section{The model and the method}

We study adsorption of hard spheres of species $S$ in a slit-like pore of the width $H$ with identical walls that have been modified by pre-adsorbed chain molecules, $C$, composed of $M$ tangentially bonded hard spheres (segments) of identical diameter $\sigma^{(C)}$. The chain connectivity is ensured by the binding potential at contact between nearest-neighbor segments. The total binding potential $V_{\mathrm{b}}$ is given by [32]:

$$
\left.\exp \left[-\beta V_{\mathrm{b}}(\mathbf{R})\right]=\prod_{i=1}^{M-1} \delta\left(\left|\mathbf{r}_{i}-\mathbf{r}_{i+1}\right|\right)-\sigma^{(C)}\right) / 4 \pi\left(\sigma^{(C)}\right)^{2}
$$

In the above $\mathbf{R}=\mathbf{r}_{\mathbf{1}}, \mathbf{r}_{\mathbf{2}}, \ldots, \mathbf{r}_{\mathbf{M}}$ denotes the vector of coordinates of all the segments, $\beta=1 / k T$.

We would like to study the model in which the first, $J=1$, and the last, $J=M$, segments of chains are "chemically" bonded to the opposite pore walls i.e. they interact with the surface via very strong, but short-ranged potentials of the form,

$$
\exp \left[-\beta v_{J}^{(C)}(z)\right]=\mathcal{C} \delta\left(\kappa_{J}\right)
$$

where $\kappa_{1}=z-\sigma^{(C)} / 2$ and $\kappa_{M}=z-\left(H-\sigma^{(C)} / 2\right)$. Here $\mathcal{C}$ is a constant, the precise value of which is irrelevant if the total number of grafted chains per unit surface area is fixed [35-39]. The external potential acting on all the remaining segments is

$$
v_{i}^{(C)}(z)= \begin{cases}\infty, & z \leqslant \sigma^{(C)} / 2, z \geqslant\left(H-\sigma^{(C)} / 2\right), \\ 0, & \text { otherwise, }\end{cases}
$$

for $i=2,3, \ldots, M-1$. It just provides the state that non-terminating segments are in the pore space between two hard walls. The total external potential acting on a chain particle is $V^{(C)}(\mathbf{R})=$ $\sum_{i=1}^{M} v_{i}^{(C)}(z)$.

Adsorption of hard spheres with the diameter $\sigma^{(S)}$ into the pore with modified walls takes place at a certain chemical potential, $\mu_{S}$. Adsorption relies on chemical equilibrium between the fluid particles in the pore and in a reservoir containing solely one-component fluid of $S$-species. During adsorption the structure of the pre-adsorbed chain molecules changes, but their amount inside the pore remains unaltered.

The fluid species $S$ interact with the wall via hard sphere potential

$$
v^{(S)}(z)= \begin{cases}\infty, & z \leqslant \sigma^{(S)} / 2, z \geqslant\left(H-\sigma^{(S)} / 2\right), \\ 0, & \text { otherwise. }\end{cases}
$$

Again, all the segments of chains and spherical molecules interact via hard sphere potentials

$$
U^{(i j)}(r)= \begin{cases}\infty, & r \leqslant \sigma^{(i j)} / 2, \\ 0, & r>\sigma^{(i j)} / 2,\end{cases}
$$

where $i, j=S, C, \sigma^{(i j)}=0.5\left(\sigma^{(i)}+\sigma^{(j)}\right)$.

In order to proceed, let us introduce the notation, $\rho^{(C)}(\mathbf{R})$ and $\rho^{(S)}(\mathbf{r})$, for the density distribution of chains and spherical species, respectively. However, the theory is constructed in terms of the density of particular segments of chains, $\rho_{s j}^{(C)}(\mathbf{r})$, that yield auxiliary quantity describing the total segment density of chains, $\rho_{s}^{(C)}(\mathbf{r})$. The segment densities are introduced via commonly used relations, see the original development in [32]

$$
\rho_{s}^{(C)}(\mathbf{r})=\sum_{j=1}^{M} \rho_{s j}^{(C)}(\mathbf{r})=\sum_{j=1}^{M} \int \mathrm{d} \mathbf{R} \delta\left(\mathbf{r}-\mathbf{r}_{j}\right) \rho^{(C)}(\mathbf{R}) .
$$


To simplify the equations below we also use the notation $\rho_{s}^{(S)}(\mathbf{r}) \equiv \rho_{s 1}^{(S)}(\mathbf{r}) \equiv \rho^{(S)}(\mathbf{r})$.

The system is studied in a grand canonical ensemble with the constraint of constancy of the number of chain molecules, i.e.

$$
R_{C}=\int \rho_{s 1}^{(C)}(z) \mathrm{d} z=\int \rho_{s M}^{(C)}(z) \mathrm{d} z
$$

where $R_{C}$ is the number of chain molecules per area of the surface. The thermodynamic potential appropriate to the description of the system is

$$
\mathcal{Y}=F\left[\rho^{(C)}(\mathbf{R}), \rho^{(S)}(\mathbf{r})\right]+\int \mathrm{d} \mathbf{r} \rho^{(S)}(\mathbf{r})\left(v^{(S)}(\mathbf{r})-\mu_{S}\right)+\sum_{i=1}^{M} \int \mathrm{d} \mathbf{r} \rho_{s i}^{(C)}(\mathbf{r}) v_{i}^{(C)}(\mathbf{r}),
$$

where $F\left[\rho^{(C)}(\mathbf{R}), \rho^{(S)}(\mathbf{r})\right]$ is the Helmholtz free energy functional.

The expression for $F\left[\rho^{(C)}(\mathbf{R}), \rho^{(S)}(\mathbf{r})\right]$ is chosen according to the theory of $\mathrm{Yu}$ and $\mathrm{Wu}[32$ ]. The density profile $\rho^{(S)}(\mathbf{r})$ and the segment density profiles $\rho_{s i}^{(C)}(\mathbf{r})$ are obtained by minimizing the functional $\mathcal{Y}$ under the constraint (7). For the sake of brevity we do not present the resulting density profile equations, (they can be found in our recent works [35 39]), but you should only note that the configurational chemical potential $\mu_{S}$ in terms of the bulk density $\rho_{b}$ of species $S$ is

$$
\mu_{S} / k T=\ln \rho_{b}+\beta \mu_{(\mathrm{hs})}^{S},
$$

where $\mu_{(\mathrm{hs})}^{S}$ is the excess chemical potential of pure hard sphere fluid at the density $\rho_{b}$ resulting here from the Carnahan-Starling equation of state.

For two planar surfaces separated by a film of adsorbed fluid, the solvation force per unit area is calculated from

$$
f_{\mathrm{s}} / k T=\frac{\partial(\mathcal{Y} / A k T)}{\partial H}-p / k T
$$

where $p$ is the pressure of the bulk system, i.e. the fluid in a reservoir. We choose the diameter of a segment of chain species as a length unit, $\sigma^{(C)}=1$. Also, for the sake of convenience the reduced solvation force as $f^{*}=f_{\mathrm{s}}\left(\sigma^{(C)}\right)^{3} / k T$ is introduced.

We would like to finish this section by the following comment. The first and the last segment of each chain are fixed at $z=\sigma^{(C)} / 2$ and $z=H-\sigma^{(C)} / 2$, respectively. The parameter $R_{C}$ determines the surface density of chains at each of the walls. Formally, the situation is symmetric with respect to the pore center for these terminating segments. However, one should remember that the terminating segments can slip within the plain separated by $\sigma^{(C)} / 2$ from the pore walls. The configuration of the first and the last segments in the $x, y$ plane is not fixed. It is formed dependent on $R_{C}$ and on the density of an adsorbed fluid. Also, the connectivity and the chain length effect this configuration. Physically, one can design a model permitting some sort of phase separation of terminating segments and adsorbed fluid species in the monolayer on the solid surface. However, we are not able to follow changes of the configuration of terminating segments in the framework of the effective one-dimensional density functional used in this study. Application of the full three-dimensional functional is not feasible numerically at present.

\section{Results and discussion}

Let us proceed to the discussion of the results. Figure 1 shows the reduced solvation force obtained for the pores with different density of grafted chains with eight segments $(M=8)$ that serve as connectors of the walls, or, in other words, form bridges between the walls in the absence of adsorbed fluid, i.e. $\rho_{b}^{*}=\rho_{b}\left(\sigma^{(C)}\right)^{3}=0$. The pore width is given everywhere below in the reduced units, $H^{*}=H / \sigma^{(C)}$. For the pores with non-modified walls the solvation force exhibits common trends. Namely it approaches zero as the wall-to-wall distance increases. Usually, for non-modified walls the solvation force, $f^{*}$, exhibits oscillatory behavior at small interparticle separations and tends to infinity for $H^{*} \rightarrow 1$. The presence of even a small amount of connecting chain molecules 
causes the state that for $H^{*} \rightarrow 8$ (i.e. when the wall-to-wall distance equals the length of extended chains) a strong attraction between the walls appears, see figure1. The plot of the reduced solvation force significantly differs from the plots shown in [30, 31]. According to the model of this study none of the bonds connecting segments of chains can be broken and the existing bridges cause the state at which the maximum value of $H$ cannot exceed $M \sigma^{(C)}$. Therefore, even for a small value of $R_{C}$ strong effective attraction between two walls appears at wall-to-wall distance close to 8 .

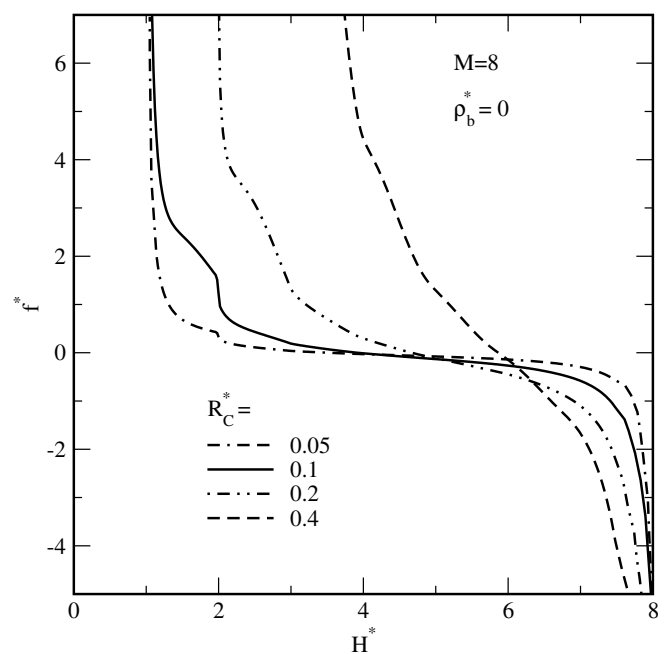

Figure 1. The reduced solvation force, $f^{*}\left(H^{*}\right)$, for the model pore with different density of grafted chains as connectors in the absence of adsorbed fluid, $\rho_{b}^{*}=0$. The calculations are for chains with eight segments $M=8$, and for the values of $R_{C}^{*}$ given in the figure.

When the number of connecting molecules (one can call them pillars) increases, then effective attraction between two walls becomes stronger at wall-to-wall separation larger than one half of the nominal chain length, c.f. the curves for $R_{C}^{*}=R_{C}\left(\sigma^{(C)}\right)^{2}=0.05$ and $R_{C}^{*}=0.2$ in figure 1 , The attractive branch for each chain density is rather smooth, i.e. we do not observe an oscillatory behavior. At small distances, strong repulsion between walls develops. For the case of a pore with non-modified walls, as well as when $R_{C}^{*}$ is small, the solvation force diverges close to $H^{*} \rightarrow 1$. However, when $R_{C}^{*}$ increases, the strong repulsion between the walls develops at larger distances, e.g. for $R_{C}^{*}=0.2$ at $H^{*} \approx 2$. Obviously, for $R_{C}^{*}$ high enough a narrow pore is almost completely filled by the chain segments and there is no room for fluid species to be adsorbed. In the absence of adsorbed fluid, one does not observe oscillations of the reduced solvation force at small distances between the walls. Rather, there is a nonmonotonous decay of the repulsion for an interval of the length $\sigma^{(C)}$, see e.g. the behavior of $f^{*}$ between $H^{*}=1$ and $H^{*}=2$ terminating with a not very sharp cusp for $R_{C}^{*}=0.1$ in figure 1. This feature actually can be attributed to the accommodation of segments of the grafted chains due to a slightly augmenting free volume when the pore width increases in this range. The cusp describing the fall of repulsion occurs when the neighbors of the grafted segment gain room for their movement in the pore space. Under such circumstances, say for $H^{*}>2$, farther segments contribute into the overall repulsion but they do not form any layer-type structure as it happens during the filling of a pore with non-modified walls by certain fluid species.

In the pores with non-modified walls, the solvation force is at maxima if the pore width approximately matches the integer number of layers of fluid species, i.e. for $n \sigma^{(S)}$, and it is at minima for $(n+1 / 2) \sigma^{(S)}$. A complementary insight into the interpretation of the solvation force can be obtained by analyzing the density profiles of chain segments given in three panels of figure 2. In the narrow pore, the segments are distributed close to the pore walls and have room for their movement only if the density of grafted chain molecules is not very high, e.g. $R_{C}^{*}=0.1$, figure 2 (a). Consequently, the solvation force is repulsive and quite high. Increasing room for chain segments to accommodate, by passing to a wider pore, $H^{*}=4$, leads to a low value of repulsive solvation force, cf. figure 2 (b) and figure 1. In another extreme, for a wide pore close to the nominal 


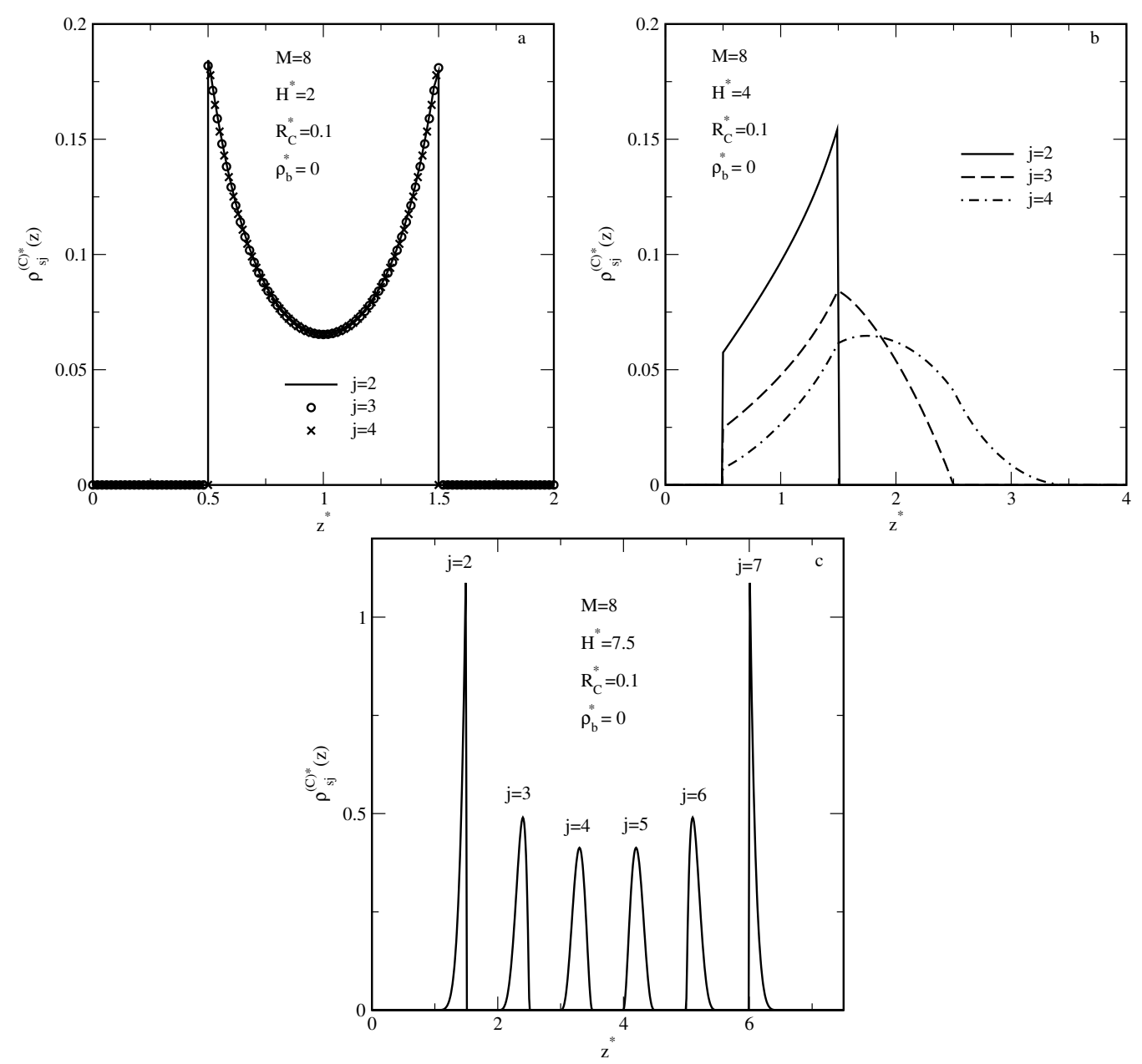

Figure 2. Density profiles of segments of chain particles, $M=8$, in the pores of different width, $H^{*}$, in the absence of adsorbed fluid, $\rho_{b}^{*}=0$, and at fixed density of grafted chains, $R_{C}^{*}=0.1$. In all panels, the index $j$ indicates the consecutive segment number. Parts (a), (b) and (c) are for $H=2, H=4$ and $H=7.5$, respectively. The nomenclature of lines is explained in the figures.

length of extended chains $H^{*}=7.5$ the segments are regularly distributed showing a layered structure. However, due to the bonding constraints accounted for in the free energy expression for the grand thermodynamic potential, the solvation force becomes attractive but without pronounced oscillations for high values of $H^{*}$, cf. figure 2 (c) and figure 1 .

The behavior of the solvation force with the bulk fluid density, $\rho_{b}^{*}$, is shown in figure 3, The amount of chain connectors is constant and not high, $R_{C}^{*}=0.1$. An increase of the density of fluid species results in more pronounced oscillations of the solvation force. However, only at the highest bulk density considered, $\rho_{b}^{*}=0.7$, the oscillations alter the repulsive branch $\left(H^{*}<5\right)$ of the curve to develop a set of attractive minima. This behavior is contrary to lower values of fluid density where the oscillations develop but still lead to repulsive solvation force. In the region of attraction, i.e. for wider pores, the solvation force is monotonous. Some density profiles of fluid species describing trends for their accommodation in layers and consequently maxima or minima of the reduced solvation force are shown in figure 4 (a). On the other hand, the corresponding profiles for the total segment density are given in figure \(b). We observe that the presence of connecting chain molecules in this case does not prohibit the development of fluid layer-like structure at high fluid density and leads to a common behavior of the solvation force for not very wide pores. These trends can be altered by increasing the density of chains or if only the fluid-segment attraction 
were present in the model.

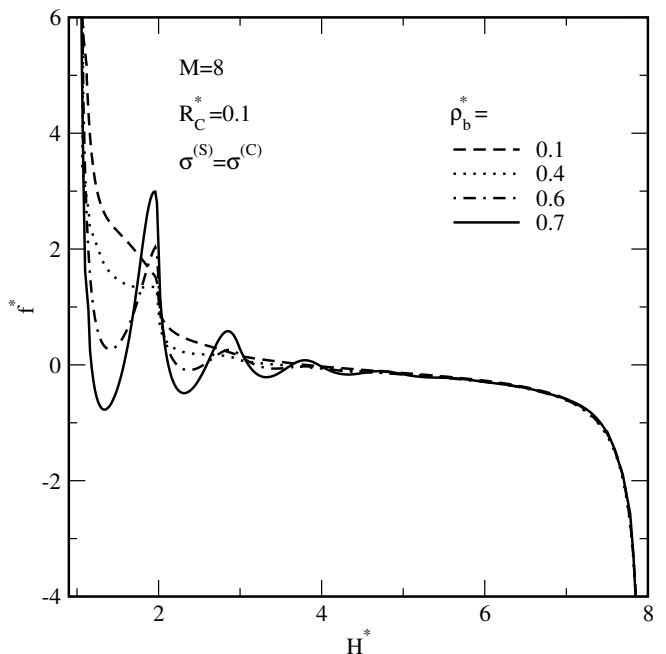

Figure 3. The reduced solvation force $f^{*}\left(H^{*}\right)$, for the model pore at different density of adsorbed fluid, $\rho_{b}^{*}$, at a fixed density of chain connectors, $R_{C}^{*}=0.1, M=8$. The nomenclature of lines is given in the figure.
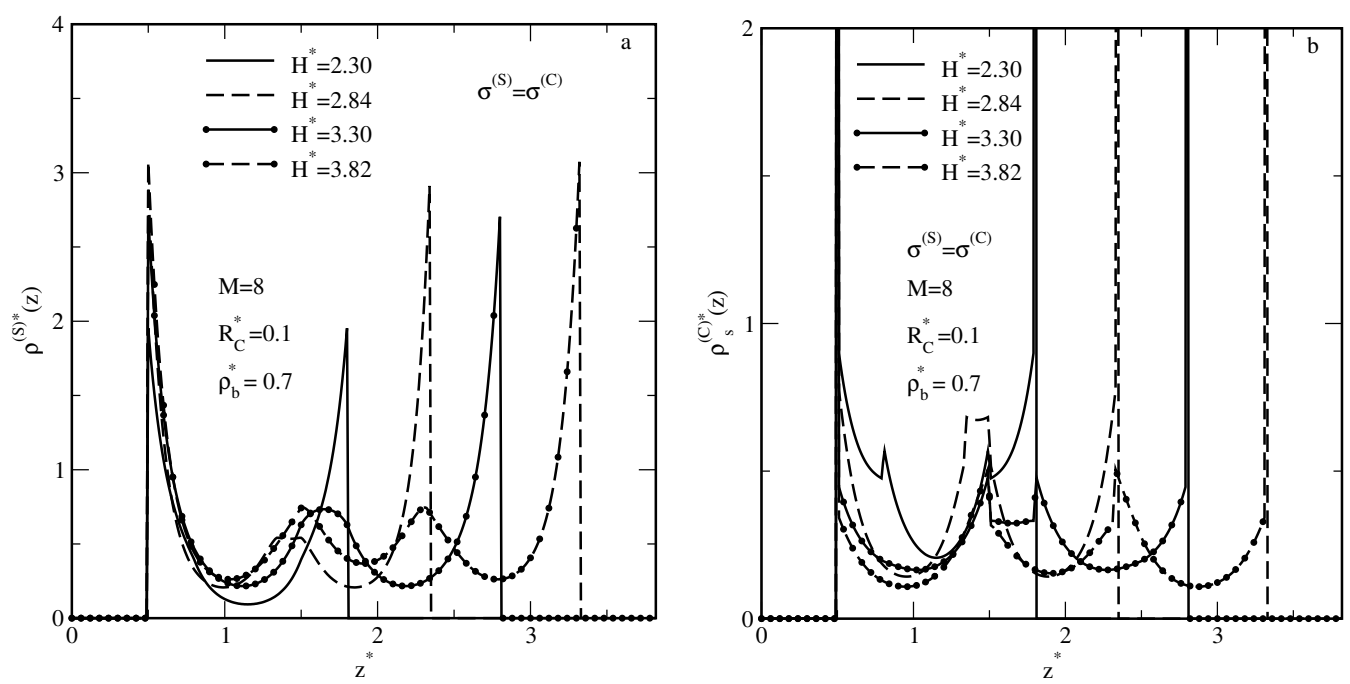

Figure 4. The density profiles of fluid species (a) and chain connectors (b) for slit-like pore of different width. The calculations are for $R_{C}^{*}=0.1, \rho_{b}^{*}=0.7$, and $M=8$. The nomenclature of lines is given in the figures.

In order to capture these possibilities at least partially, we have performed calculations at a constant high fluid density and varied the amount of chain connectors. Moreover, for the sake of generality, we would like to get insight into the effects of fluid species and of chain segments on the oscillations of the solvation force separately by considering the model with unequal diameters. In the restricted case of equal diameters two species may contribute to the solvation force at the same distance scale. First, we studied the case in which fluid species have slightly smaller diameter than the segments of chains, i.e. $\sigma^{(S)}=0.8 \sigma^{(C)}$. One set of results concerning such a model is given in figure 5. The fluid density is chosen such to correspond to 0.7, if the diameters of segments and fluid particles are equal. From this figure we learn that the scale of oscillations of the solvation force is determined by the diameter of fluid species. Most importantly, we observe that 
the attractive branch of the solvation force becomes oscillatory, if the density of connector chain molecules is sufficiently high. This chain density should provide an overall attraction between pore walls whereas the fluid density should be high enough to provide the formation of layers of fluid species. Then, the total picture is like the one we show in figure 5. The density profiles illustrating distribution of both species in the pore are shown in figure 6 ( $a$ and b).

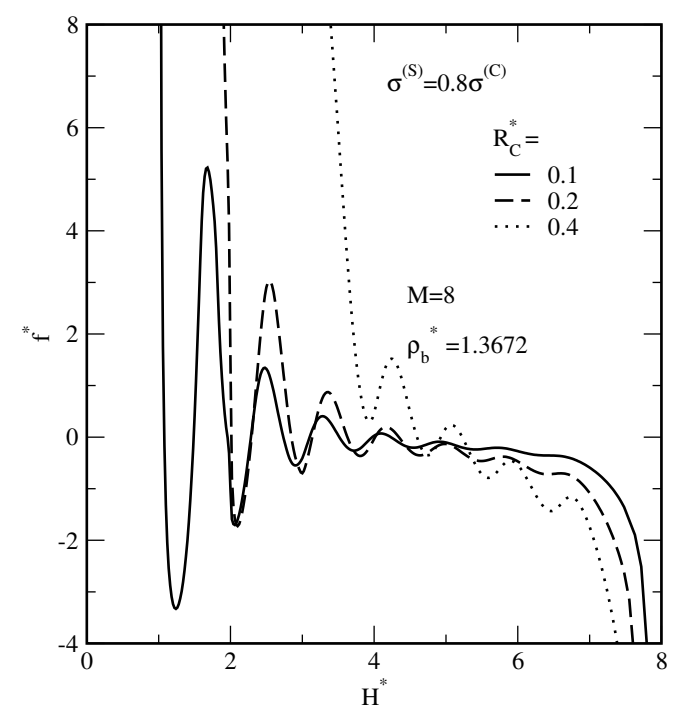

Figure 5. The reduced solvation force as a function of the density of chain connectors $R_{C}^{*}$ $(M=8)$ at a fixed bulk fluid density, $\rho_{b}^{*}=1.3672$. The fluid particles are smaller than chain segments, $\sigma^{(S)}=0.8 \sigma^{(C)}$.
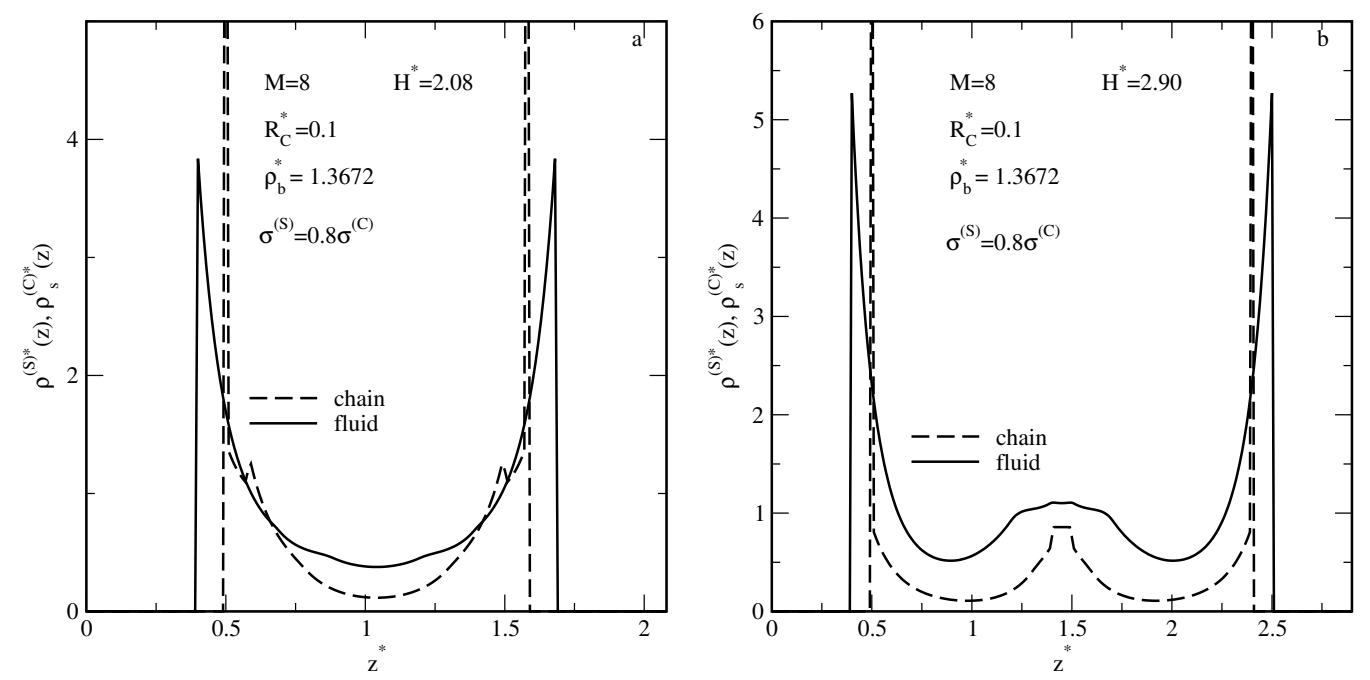

Figure 6. The density profiles of fluid species $\rho^{(S)^{*}}(z)$ and the total segment density profiles $\rho_{s}^{(C)^{*}}(z)$ (panels a and b) for selected pore width, $H^{*}$ as an illustration of the behavior of the solvation force shown in figure 5. The nomenclature of lines is given in the figures.

If the fluid particles are of larger diameter than the segments of chain connectors, the behavior of the reduced solvation force differs from what was discussed concerning similar curves in figure 5 . Some examples of the solvation force for the model in which $\sigma^{(S)}=1.6 \sigma^{(C)}$ are given in figure 7 (a). The bulk fluid density is chosen to coincide with 0.7 , if the diameters of segments and fluid particles were equal. In this case we observe that the solvation force behaves similarly to the model without 
fluid species, cf. figure 1, if the density of chain pillars is not high, e.g. $R_{C}^{*}=0.1$ and $R_{C}^{*}=0.2$. At a higher density of chain connectors, $R_{C}^{*}=0.4$, the attractive branch becomes just slightly oscillatory indicating some sort of ordering of fluid species in wider pores. In the intermediate pore, $H^{*}=4$, that cannot accommodate an integer number of fluid layers, the solvation force is weakly attractive, and the distribution of species in such a pore can be imagined by analyzing the density profiles shown in figure 7 (b).
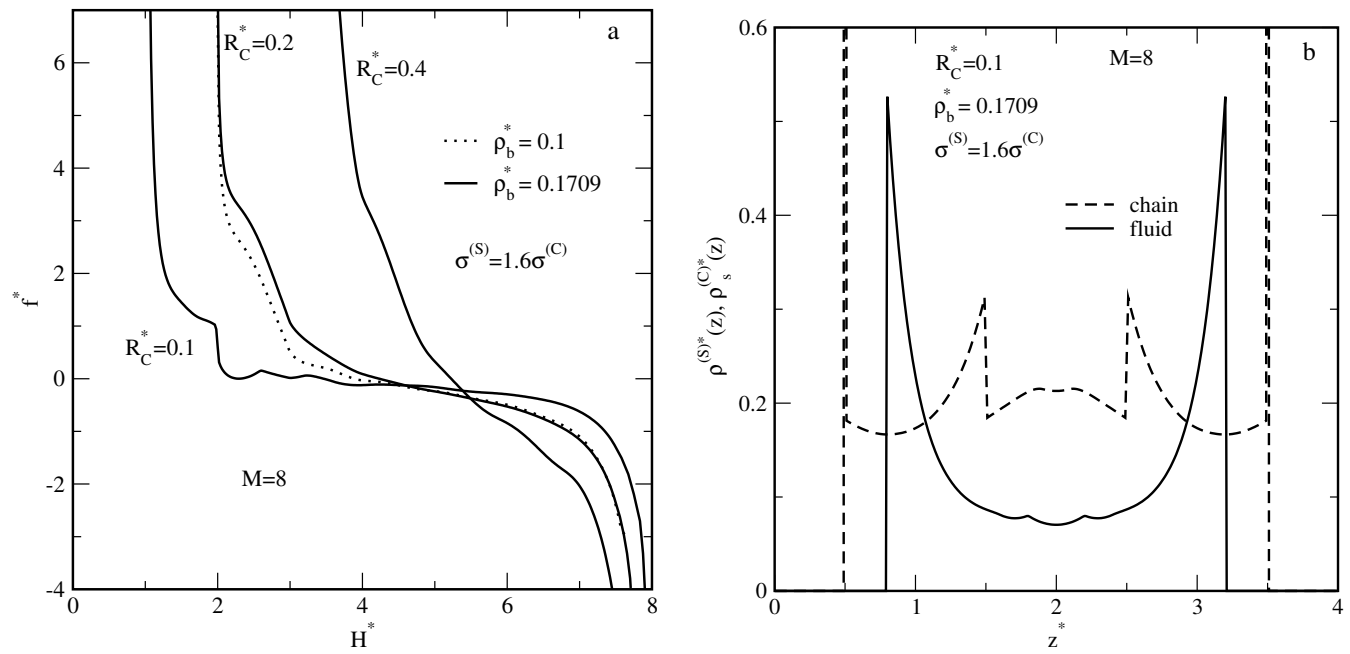

Figure 7. (a) The reduced solvation force as a function of the density of chain connectors, $R_{C}^{*}$, at a fixed bulk fluid density, $\rho_{b}^{*}=0.1709$. The fluid particles are of larger diameter than chain segments, $\sigma^{(S)}=1.6 \sigma^{(C)}$. (b) The density profiles of fluid species $\rho^{(S)^{*}}(z)$ and the total segment density profiles $\rho_{s}^{(C)^{*}}(z)$ for $H^{*}=4$ as an illustration of the behavior of the solvation force shown in (a).

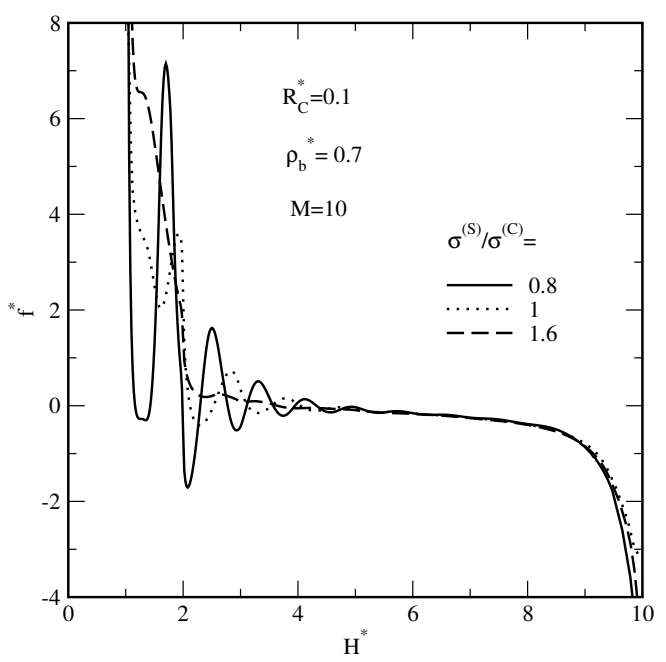

Figure 8. A comparison of the reduced solvation force for the models characterized by the different ratio of diameters of fluid species and chain segments.

A summarizing insight into the effects of size ratio of segments and fluid particles at low density of chain connectors with $M=10$ and fixed reduced density of the bulk fluid, $\rho_{b}^{*}$, is presented in figure 8. The fragment of the solvation force curves corresponding to rather narrow pores is very sensitive to the type of fluid one adsorbs into the pores. Smaller species more easily develop a layer-type structure which is manifested in the oscillations of the solvation force. Only for a set of 
specific pore widths one can find either repulsive maxima or attractive minima in the adsorption of particular fluid species. Another important factor effecting the adsorption of a given fluid into narrow pores is the bulk fluid density or equivalently the chosen value of the fluid chemical potential in the bulk reservoir (or pressure), figure 9 (a). For wider pores, the value of an attractive force hardly depends on fluid density and is predominantly determined by the density of chain connectors. Thus, there exists a certain window of parameters of fluid density, chain pillars density and the number of segments in chains that permit to manipulate adsorption and the solvation force most efficiently for pores of a given width.
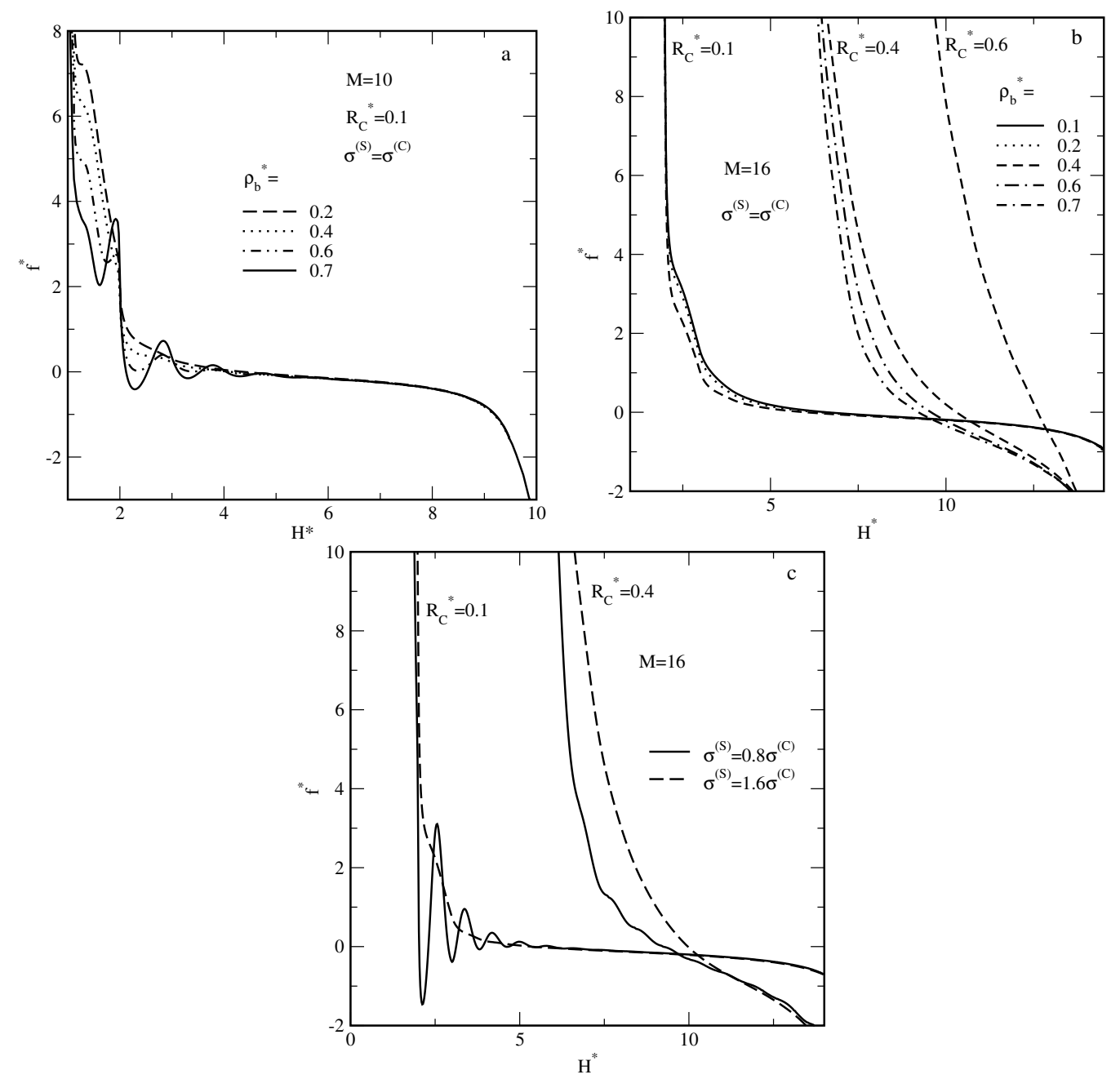

Figure 9. (a) The reduced solvation force $f^{*}\left(H^{*}\right)$, for the model pore at different density of adsorbed fluid, $\rho_{b}^{*}$, at a fixed density of chain connectors, $R_{C}^{*}=0.1, M=10$. The nomenclature of lines is given in the figure. (b) The same as in (a), but for the model with $M=16$. (c) The same as in figure 8 but for the model with $M=16, \rho_{b}^{*}=0.7$.

Our previous discussion has been focused on rather short chains $M=8$ and $M=10$. We have not studied much longer chains so far. However, for the sake of better illustration and to confirm our previous discussion, we give only two results concerning the effect of bulk fluid density and of size ratio together with the effect of chain pillars density in figures 9 (b) and 9 (c) for chains with sixteen segments, $M=16$. These figures confirm our above mentioned conclusions and in some sense provide estimates for the range of parameters that are necessary to be chosen in order to effect the entropic solvation force between pore walls as desired.

A brief summary of this study is as follows. We have investigated the entropic solvation forces 
induced by short polymers serving as connectors of the pore walls (pillars) via a density functional approach. Our focus was on the origin of attraction/repulsion in these systems. The pillars could not be broken, therefore at the wall-to-wall separation approaching the pillar length we observed the development of a strong, diverging attraction. On the other hand, in narrow pores an overall repulsion is observed. Both parts of the solvation force curve can be modulated in order to yield an oscillatory behavior under a certain choice of the density of the pillars, their length (number of segments), external pressure for desired adsorbing conditions for specific fluid systems. It seems that there exists a window of the values of parameters permitting to manipulate the solvation force most efficiently. Some possible extensions of the present study have been already noted in the introduction. Nevertheless, it is important to mention that additional theoretical and computer simulation work is necessary to clearly understand the complex systems of this study.

\section{Acknowledgements}

O.P. has been supported by the grant IN223808 of the National University of Mexico. S.S. acknowledges partial support from the grant Number N N204 123737 of the Ministry of Science of Poland.

\section{References}

1. Israelachvili J.N., Intermolecular and Surface Forces, 2nd ed. Academic, London, 1991.

2. Israelachvili J.N., Adams G.E., J. Chem. Soc. Faraday Trans. I, 1978, 74, 975.

3. Demirel L.A., Granick S., J. Chem. Phys., 2001, 115, 1490.

4. Israelachvili J.N., Acc. Chem. Res., 1987, 20, 415.

5. Israelachvili J.N., Wennerström H., J. Phys. Chem., 1992, 96, 520.

6. Attard P., Parker J.L., J. Phys. Chem., 1992, 96, 5086.

7. Cevc G., J. Chem. Soc., Faraday Trans. I, 1991, 87, 2733.

8. Wang Y., Hill K., Harris J.G., Langmuir, 1993, 9, 1983.

9. Dijkstra M., J. Chem. Phys., 1997, 107, 3277.

10. Zhou S., Chem. Phys. Lett., 2004, 392, 110.

11. Roth R., Evans R., Dietrich S., Phys. Rev. E, 2000, 62, 5360.

12. Chen Y.L., Schweizer K.S., J. Chem. Phys., 2002, 117, 1351.

13. Qin Y., Fichthorn K.A., J. Chem. Phys., 2007, 127, 144911.

14. Di Leo J.M., Marañón J., J. Mol. Struct.: Theochem., 2003, 623, 159.

15. Shinto H., Uranishi K., Miyahara M., Higashitani K., J. Chem. Phys., 2002, 116, 9500.

16. Porcheron F., Rousseau B., Schoen M., Fuchs A.H., Phys. Chem. Chem. Phys., 2001, 3, 1155.

17. Frink L.J.D., van Swol F., J. Chem. Phys., 1998, 108, 5588.

18. Luo J., Qian L., Wen S., Wen L., Wen S., Li L.K.Y., Tribology Trans., 1999, 42, 912.

19. Ghatak C., Ayappa K.G., J. Chem. Phys., 2004, 120, 9703.

20. Henderson J.R., Molec. Simul., 2005, 31, 435.

21. Sprenger M., Schlesener F., Dietrich S., J. Chem. Phys., 2006, 124, 134703.

22. Noy A., Frisbie C.D., Rozsnyai L.F., Wrighton M.S., Lieber C.M., J. Am. Chem. Soc., 1995, 117, 7943.

23. Hackett E., Manias E., Giannelis E.P., J. Chem. Phys., 1998, 108, 7410.

24. Raffaini G., Ganazzoli F., J. Mat. Sci.: Materials in Medicine, 2007, 18, 309.

25. Zhao B., Brittain W.J., Prog. Pol. Sci., 2000, 25, 677.

26. Clear S.C., Nealey P.F., J. Chem. Phys., 2001, 114, 2802.

27. Wu J.Z., AIChE J., 2006, 52, 1169.

28. Wu J.Z., Li Z., Ann. Rev. Phys. Chem., 2007, 58, 85.

29. Fu D., J. Chem. Phys., 2006, 124, 164701.

30. Cao D., Wu J.Z., Langmuir, 2006, 22, 2712.

31. Jiang T., Li Z., Wu J.Z., Macromolecules, 2007, 40334.

32. Yu Y.X., Wu J., J. Chem. Phys., 2002, 117, 2368.

33. Pizio O., Pusztai L., Sokołowska Z., Sokołowski S., J. Chem. Phys., 2009, 130, 134501.

34. Xu X., Cao D., J. Chem. Phys., 2009, 130, 164901.

35. Borówko M., Rżysko W., Sokołowski S., Staszewski T., J. Chem. Phys., 2007, 126, 214703.

36. Matusewicz M., Patrykiejew A., Sokołowski S., Pizio O., J. Chem. Phys., 2007, 127, 174707. 
37. Pizio O., Borówko M., Rżysko W., Staszewski T., Sokołowski S., J. Chem. Phys., 2008, 128, 044702.

38. Sokołowska Z., Sokołowski S., J. Coll. Interface Sci., 2007, 316, 652.

39. Patrykiejew A., Sokołowski S., Tscheliessnig R., Fischer J., Pizio O., J. Phys. Chem. B., 2008, 112, 4552.

40. Pizio O., Bucior K., Patrykiejew A., Sokołowski S., J. Chem. Phys., 2005, 123, 214902.

\title{
Ентропійна сила сольватації між поверхнями, модифікованими розгалуженими ланцюжками: метод функціоналу густини
}

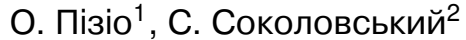 \\ 1 Інститут хімії УНАМ, Койокан 04510, Мексика \\ 2 Університет Марії Склодовської-Кюрі, Люблін, Польща
}

\begin{abstract}
Поведінка плину твердих сфер у щілиноподібних порах зі стінками, модифікованими розгалуженими ланцюговими молекулами, які складаються із твердосферних сегментів, досліджується із використанням теорії функціоналу густини. Ланцюги є розгалуженими до протилежних стінок через скінчені сегменти, що формують опори колони. Детально досліджено вплив обмеження і "хімічної" модифікації стінок пори на ентропійну силу сольватації. Ми спостерігаємо, що при відсутності адсорбованого плину сила сольватації є сильно відштовхувальною для вузьких пор і притягальною для широких пор. Коли адсорбований плин є присутнім, обидві частини кривої сили сольватації можуть розвивати осциляційну поведінку в залежності від густини опор колон, числа сегментів і умов адсобції. Також, розмірний коефіцієнт між частинками адсорбованого плину і сегментами ланцюгів $€$ важливим для розвитку осциляцій. Вибір цих параметрів $€$ дуже суттєвим для ефективного керування силою сольватації для пор різної ширини.
\end{abstract}

Ключові слова: метод функціоналу густини, розгалужені ланцюжки, сила сольватації, профілі густини 